\title{
Signet ring cell carcinoma of the stomach: correlation between endocytoscopy and histology
}

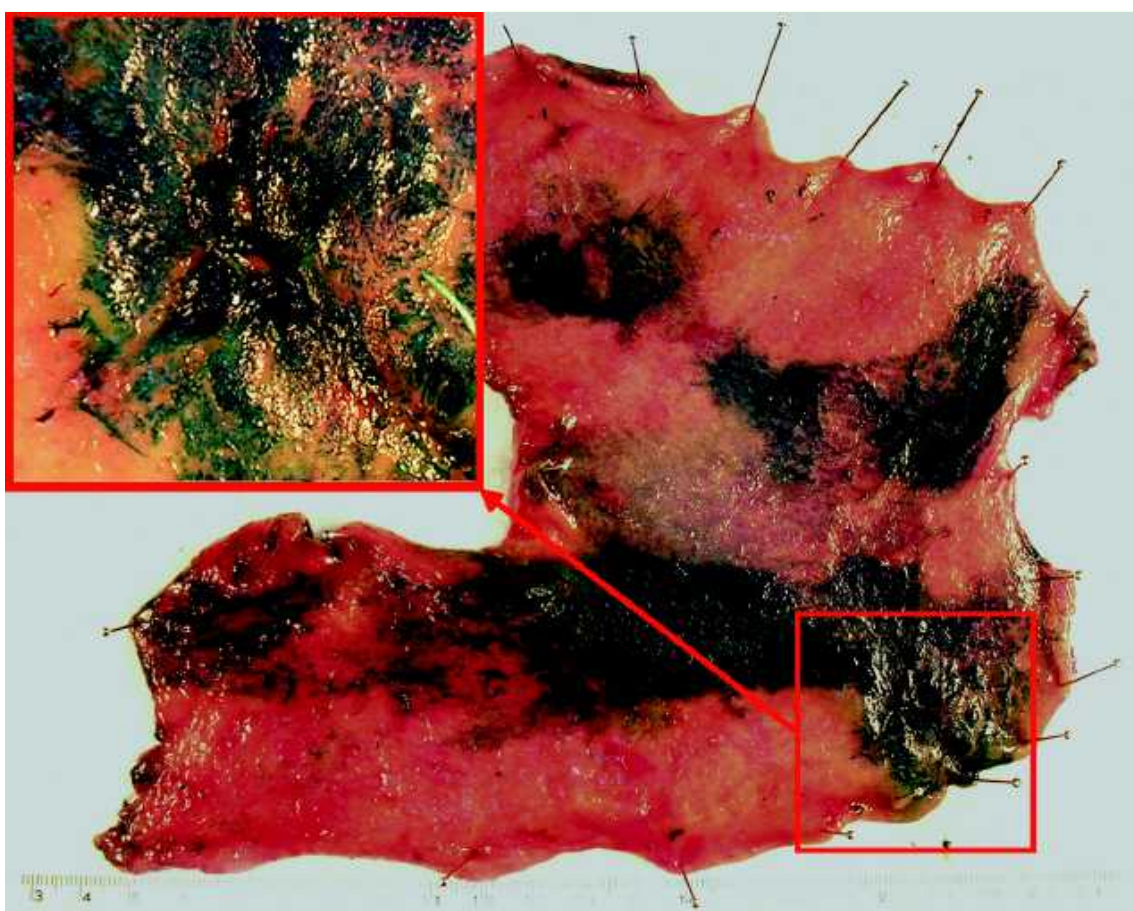

Fig. 1 Fresh surgical specimen showing signet ring cell early gastric cancer located at the anterior wall of the gastric antrum. Magnified image (box) refers to lesion details. The specimen has already been stained using methylene blue for endocytoscopy observation.

Our experience with endocytoscopy (ECS) in the lower gastrointestinal tract has shown good correlation between ECS and histology in detecting aberrant crypt foci [1] and carcinoid tumor [2]. To our knowledge, ECS imaging of gastric signet ring cell (SRC) carcinoma has not been previously described.

A 40-year-old man and a 60-year-old woman underwent subtotal gastrectomy at our institution for early gastric cancer, which was histologically proven to be SRC carcinoma. Previous endoscopic examination had classified the lesions as type 0 IIa-c and type 0 III [3], with diameters of $1.3 \mathrm{~cm}$ and $1.5 \mathrm{~cm}$, respectively. In both cases macroscopic examination of fresh surgical specimens confirmed the presence of a nodule at the gastric anterior wall ( Fig. 1). After staining according to the technique already described $[1,2]$, lesions were evaluated using a prototype Olympus XEC-300-U endocytoscope (Olympus Medical System Co., Tokyo, Japan).

No glandular structures were observed on ECS examination; instead these structures had been replaced by groups of cellular elements characterized by cytoplasmic halos and a peripheral location of the nucleus ( Fig. 2 a). This finding corresponded to the typical histologic aspect of SRC carcinoma, having a large amount of mucin within neoplastic cells, which was confirmed by both standard hematoxylin and eosin histologic examination ( $\bullet$ Fig. $\mathbf{2 b}$ ) and immunophenotypical characterization of the areas previously explored by ECS. The morphologic correspondence between ECS images ( $\bullet$ Fig. 3 a) and histology ( $\bullet$ Fig. 3b) was also striking for single peculiar neoplastic cells.

If these results are confirmed by further experience in vivo, ECS could gain a relevant role in the therapeutic decisionmaking process for early gastric cancer, which requires precise information, including tumor differentiation and size [4]. The ability to target bioptic sampling by means of ECS for both lesion and the transition zone between cancer and normal mucosa, might improve the selection of patients for endoscopic mucosal resection.

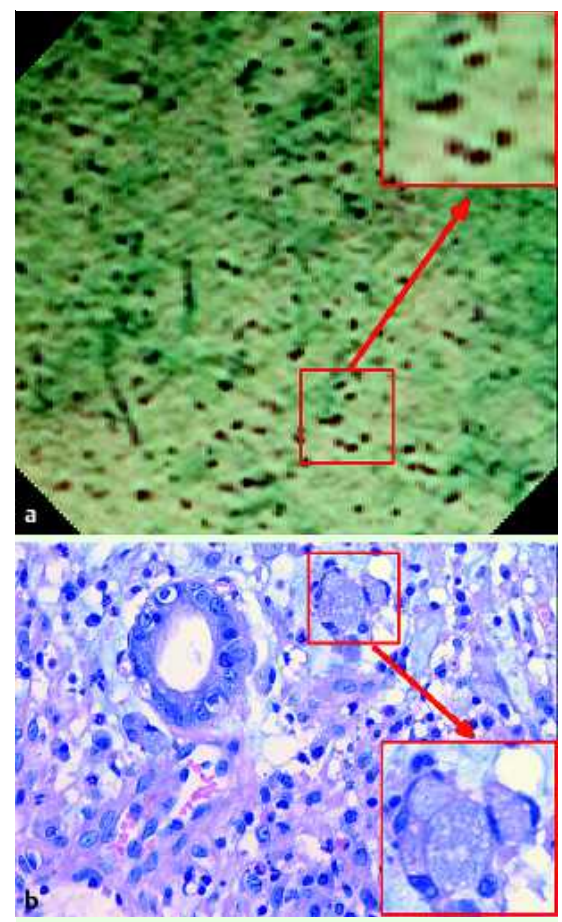

Fig. 2 a Endocytoscopic $(\times 450)$ picture of signet ring cell carcinoma showing a group of neoplastic cells (magnified details within the box), with a cytoplasmic halo and peripheral nucleus. b Histopathology (hematoxylin and eosin $[H \& E] \times 400$ ) correlates well with the endocytoscopy image. Nuclei are located peripherally due to the large amount of intracellular mucin.



Fig. 3 a Endocytoscopic ( $\times 450)$ image of signet ring cell carcinoma showing typical appearance of tumor cells (magnified within the box). $\mathbf{b}$ Histopathologic $(H \& E, \times 400)$ aspect of a single signet ring cell corresponding to endocytoscopy image. 
Endoscopy_UCTN_Code_CCL_1AB_2AD_3AB

\section{A. Fasoli ${ }^{1}$, V. Pugliese ${ }^{1}$, M. Furnari ${ }^{1}$,}

B. Gatteschi ${ }^{2}$, M. Truini ${ }^{2}$, E. Meroni ${ }^{1}$

1 Division of Interdisciplinary Endoscopy, National Institute for Cancer Research, Genoa, Italy

2 Division of Pathology, National Institute for Cancer Research, Genoa, Italy

\section{References}

1 Meroni E, Gatteschi B, Fasoli A et al. Detection of tissue abnormalities in normal mucosa surrounding colorectal cancer using an endocytoscopy system. Endoscopy 2007; 39: 369- 370

2 Fasoli A, Pugliese V, Gatteschi B et al. Endocytoscopic imaging of a carcinoid tumor. Gastrointest Endosc 2008; 68: 1015 - 1017

3 Endoscopic Classification Review Group. Update on the Paris classification of superficial neoplastic lesions in the digestive tract. Endoscopy 2005; 37: 570-578

4 National Comprehensive Cancer Network. Clinical Practice Guidelines in Oncology. Gastric cancer. Fort Washington, PA, 2008: Version 1. 2009
Bibliography

DOI $10.1055 / \mathrm{s}-0028-1119457$

Endoscopy 2009; 41: E65-E66

(c) Georg Thieme Verlag KG Stuttgart · New York . ISSN 0013-726X

\section{Corresponding author}

\section{E. Meroni, MD}

Division of Interdisciplinary Endoscopy National Institute for Cancer Research 10 Largo R. Benzi

16132 Genoa

Italy

Fax: +39-010-5600645

emanuele.meroni@istge.it 